\title{
Generic Dynamic Scaling in Kinetic Roughening
}

\author{
José J. Ramasco, ${ }^{1,2, *}$ Juan M. López, ${ }^{3, \dagger}$ and Miguel A. Rodríguez ${ }^{1}$ \\ ${ }^{1}$ Instituto de Física de Cantabria, CSIC-UC, E-39005 Santander, Spain \\ ${ }^{2}$ Departamento de Física Moderna, Universidad de Cantabria, E-39005 Santander, Spain \\ ${ }^{3}$ Dipartamento di Fisica and Unità INFM, Università di Roma "La Sapienza", I-00185 Roma, Italy
}

(Received 12 July 1999)

\begin{abstract}
We study the dynamic scaling hypothesis in invariant surface growth. We show that the existence of power-law scaling of the correlation functions (scale invariance) does not determine a unique dynamic scaling form of the correlation functions, which leads to the different anomalous forms of scaling recently observed in growth models. We derive all the existing forms of anomalous dynamic scaling from a new generic scaling ansatz. The different scaling forms are subclasses of this generic scaling ansatz associated with bounds on the roughness exponent values. The existence of a new class of anomalous dynamic scaling is predicted and compared with simulations.
\end{abstract}

PACS numbers: 68.35.Fx, 05.40.-a, 05.70.Ln

The theory of kinetic roughening deals with the fate of surfaces growing in nonequilibrium conditions [1,2]. In a typical situation an initially flat surface grows and roughens continuously as it is driven by some external noise. The noise term can be of thermal origin (such as, for instance, fluctuations in the flux of particles in a deposition process) or a quenched disorder (such as in the motion of driven interfaces through porous media). A rough surface may be characterized by the fluctuations of the height around its mean value. So, a basic quantity to look at is the global interface width, $W(L, t)=\left\langle\overline{[h(x, t)-\bar{h}]^{2}}\right\rangle^{1 / 2}$, where the overbar denotes average over all $x$ in a system of size $L$ and the brackets denote average over different realizations. Rough surfaces then correspond to situations in which the stationary width $W(L, t \rightarrow \infty)$ grows with the system size. Alternatively, one may calculate other quantities related to correlations over a distance $l$ as the height-height correlation function, $G(l, t)=\left\langle\overline{[h(x+l, t)-h(x, t)]^{2}}\right\rangle$, or the local width, $w(l, t)=\left\langle\left\langle\left[h(x, t)-\langle h\rangle_{l}\right]^{2}\right\rangle_{l}\right\rangle^{1 / 2}$, where $\langle\cdots\rangle_{l}$ denotes an average over $x$ in windows of size $l$.

In the absence of any characteristic length in the problem growth processes are expected to show power-law behavior of the correlation functions in space and time, and the Family-Vicsek dynamic scaling ansatz [1-3],

$$
W(L, t)=t^{\alpha / z} f(L / \xi(t)),
$$

ought to hold. The scaling function $f(u)$ behaves as

$$
f(u) \sim \begin{cases}u^{\alpha} & \text { if } u \ll 1 \\ \text { const } & \text { if } u \gg 1,\end{cases}
$$

where $\alpha$ is the roughness exponent and characterizes the stationary regime, in which the horizontal correlation length $\xi(t) \sim t^{1 / z}$ ( $z$ is the so called dynamic exponent) has reached a value larger than the system size $L$. The ratio $\beta=\alpha / z$ is called growth exponent and characterizes the short time behavior of the surface. As occurs in equilibrium critical phenomena, the corresponding critical exponents do not depend on microscopic details of the system under investigation. This has made it possible to divide growth processes into universality classes according to the values of these characteristic exponents $[1,2]$.

A most intriguing feature of some growth models is that the above standard scaling of the global width differs substantially from the scaling behavior of the local interface fluctuations (measured either by the local width or the height-height correlation). More precisely, in some growth models the local width (and the height-height correlation) scales as in Eq. (1), i.e., $w(l, t)=t^{\beta} f_{A}(l / \xi(t))$, but with the anomalous scaling function

$$
f_{A}(u) \sim \begin{cases}u^{\alpha_{\mathrm{loc}}} & \text { if } u \ll 1 \\ \text { const } & \text { if } u \gg 1\end{cases}
$$

where the new independent exponent $\alpha_{\text {loc }}$ is called the local roughness exponent. This is what has been called anomalous roughening in the literature, and has been found to occur in many growth models [4-10] as well as experiments [11-15]. Moreover, it has recently been shown $[16,17]$ that anomalous roughening can take two different forms. On the one hand, there are super-rough processes, i.e., $\alpha>1$, for which always $\alpha_{\text {loc }}=1$. On the other hand, there are intrinsically anomalous roughened surfaces, for which $\alpha_{\text {loc }}<1$ and $\alpha$ can actually be any $\alpha>\alpha_{\text {loc }}$.

Anomalous scaling implies that one more independent exponent, $\alpha_{\text {loc }}$, may be needed in order to assess the universality class of the particular system under study. In other words, some growth models may have exactly the same $\alpha$ and $z$ values seemingly indicating that they belong to the same universality class. However, they may have different values of $\alpha_{\text {loc }}$ showing that they actually belong to distinct classes of growth. As for the experiments, only the local roughness exponent is measurable by direct methods, since the system size remains normally fixed. Fracture experiments [14] in systems of varying sizes have succeeded in measuring both the local and global roughness exponents, in good agreement with the scaling picture described above.

In this Letter we introduce a new anomalous dynamics in kinetic roughening. We show that, by adopting more 
general forms of the scaling functions involved, a generic theory of dynamic scaling can be constructed. Our theory incorporates all the different forms that dynamic scaling can take, namely, Family-Vicsek, super-rough, and intrinsic, as subclasses and predicts the existence of a new class of growth models with novel anomalous scaling properties. Simulations of the Sneppen model (rule A) [18] of selforganized depinning (and other related models) are presented as examples of the new dynamics.

First, let us consider the Fourier transform of the height of the surface in a system of size $L$, which is given by $\hat{h}(k, t)=L^{-1 / 2} \sum_{x}[h(x, t)-\bar{h}(t)] \exp (i k x)$, where the spatial average of the height has been subtracted. The scaling behavior of the surface can now be investigated by calculating the structure factor or power spectrum,

$$
S(k, t)=\langle\hat{h}(k, t) \hat{h}(-k, t)\rangle,
$$

which is related to the height-height correlation function $G(l, t)$ defined above by

$$
\begin{aligned}
G(l, t)= & \frac{4}{L} \sum_{2 \pi / L \leq k \leq \pi / a}[1-\cos (k l)] S(k, t) \\
& \propto \int_{2 \pi / L}^{\pi / a} \frac{d k}{2 \pi}[1-\cos (k l)] S(k, t),
\end{aligned}
$$

where $a$ is the lattice spacing and $L$ is the system size.

In order to explore the most general form that kinetic roughening can take, we study the scaling behavior of surfaces satisfying what we will call a generic dynamic scaling form of the correlation functions. We will consider that a growing surface satisfies a generic dynamic scaling when there exists a correlation length $\xi(t)$, i.e., the distance over which correlations have propagated up to time $t$, and $\xi(t) \sim t^{1 / z}, z$ being the dynamic exponent. If no characteristic scale exists but $\xi$ and the system size $L$, then power-law behavior in space and time is expected and the growth saturates when $\xi \sim L$ and the correlations [and from Eq. (5) also the structure factor] become time-independent. The global roughness exponent $\alpha$ can now be calculated in this regime from $G\left(l=L, t \gg L^{z}\right) \sim L^{2 \alpha}$ [or $\left.W\left(L, t \gg L^{z}\right) \sim L^{\alpha}\right]$. In general, as we will see below, the scaling function that enters the dynamic scaling of the local width (or the height-height correlation) takes different forms depending on further restrictions and/or bounds for the roughness exponent values. These kinds of restrictions are very often assumed and not valid for every growth model. For instance, only if the surface were self-affine, saturation of the correlation function $G(l, t)$ would also occur for intermediate scales $l$ at times $t \sim l^{z}$ and with the very same roughness exponent. However, the latter does not hold when anomalous roughening takes place, as can be seen from the scaling of the local width in Eq. (3).

Our aim here is to investigate all possible forms that the scaling functions can exhibit when solely the existence of generic scaling is assumed. So, if the roughening process under consideration shows generic dynamic scaling (in the sense explained above), and no further assumptions (such as, for instance, surface self-affinity or implicit bounds for the exponent values) are imposed, then we propose that the structure factor is given by

$$
S(k, t)=k^{-(2 \alpha+1)} s\left(k t^{1 / z}\right),
$$

where the scaling function has the general form

$$
s(u) \sim \begin{cases}u^{2\left(\alpha-\alpha_{s}\right)} & \text { if } u \gg 1 \\ u^{2 \alpha+1} & \text { if } u \ll 1,\end{cases}
$$

and the exponent $\alpha_{s}$ is what we will call the spectral roughness exponent. This scaling ansatz is a natural generalization of the scaling proposed for the structure factor in Refs. [16,17] for anomalous scaling.

In the case of the global width, one can make use of

$$
W^{2}(L, t)=\frac{1}{L} \sum_{k} S(k, t)=\int \frac{d k}{2 \pi} S(k, t),
$$

to prove easily that the global width scales as in Eqs. (1) and (2), independently of the value of the exponents $\alpha$ and $\alpha_{s}$.

However, the scaling of the local width is much more involved. The existence of a generic scaling behavior such as (7) for the structure factor always leads to a dynamic scaling behavior,

$$
w(l, t) \sim \sqrt{G(l, t)}=t^{\beta} g(l / \xi),
$$

of the height-height correlation (and local width), but the corresponding scaling function $g(u)$ is not unique. When substituting Eqs. (7) and (6) into (5), one can see that the various limits involved $[a \rightarrow 0, \xi(t) / L \rightarrow \infty$, and $L \rightarrow$ $\infty]$ do not commute $[16,17]$. This results in a different scaling behavior of $g(u)$ depending on the value of the exponent $\alpha_{s}$.

Let us now summarize how all scaling behaviors reported in the literature are obtained from the generic dynamic scaling ansatz (7). We shall also show how a new roughening dynamics naturally appears in this scaling theory. Two major cases can be distinguished, namely, $\alpha_{s}<1$ and $\alpha_{s}>1$. On the one hand, for $\alpha_{s}<1$ the integral in Eq. (5) has already been computed $[16,17]$ and one gets

$$
g_{\alpha_{s}<1}(u) \sim\left\{\begin{array}{ll}
u^{\alpha_{s}} & \text { if } u \ll 1 \\
\text { const } & \text { if } u \gg 1
\end{array} .\right.
$$

So, the corresponding scaling function is $g_{\alpha_{s}<1} \sim f_{A}$ and $\alpha_{s}=\alpha_{\text {loc }}$, i.e., the intrinsic anomalous scaling function in Eq. (3). Moreover, in this case the interface would satisfy a Family-Vicsek scaling (for the local as well as the global width) only if $\alpha=\alpha$ sere satisfied for the particular growth model under study. Thus, the standard Family-Vicsek scaling turns out to be one of the possible scaling forms compatible with generic scaling invariant growth, but not the only one.

On the other hand, a new anomalous dynamics shows up for growth models in which $\alpha_{s}>1$. In this case, one 
finds that, in the thermodynamic limit $L \rightarrow \infty$, the integral Eq. (5) has a divergence coming from the lower integration limit. To avoid the divergence one has to compute the integral keeping $L$ fixed. We then obtain the scaling function

$$
g_{\alpha_{s}>1}(u) \sim \begin{cases}u & \text { if } u \ll 1 \\ \text { const } & \text { if } u \gg 1\end{cases}
$$

So in this case one always gets $\alpha_{\text {loc }}=1$ for any $\alpha_{s}>1$. Thus, for growth models in which $\alpha=\alpha_{s}$, one recovers the super-rough scaling behavior $[16,17]$.

However, it is worth noting that neither the spectral exponent $\alpha_{s}$ nor the global exponent $\alpha$ are fixed by the scaling in Eqs. (7) and (11) and, in principle, they could be different. Therefore, growth models in which $\alpha_{s}>1$ but $\alpha \neq \alpha_{s}$ could also be possible and represent a new type of dynamics with anomalous scaling. The main feature of this new type of anomalous roughening is that it can be detected only by determining the scaling of the structure factor. Whenever such a scaling takes place in the problem under investigation the new exponent $\alpha_{s}$ will show up only when analyzing the scaling behavior of $S(k, t)$ and will not be detectable in either $W(L, t), w(l, t)$, or $G(l, t)$. In fact, as we have shown, the stationary regime of a surface exhibiting this kind of anomalous scaling will be characterized by $W(L) \sim L^{\alpha}$ and $w(l, L) \sim \sqrt{G(l, L)} \sim l L^{\alpha-1}$; however, the structure factor scales as $S(k, L) \sim k^{-\left(2 \alpha_{s}+1\right)} L^{2\left(\alpha-\alpha_{s}\right)}$, where the spectral roughness exponent $\alpha_{s}$ is a new and independent exponent. We can summarize our analytical results as follows:

$$
\begin{cases}\text { if } \alpha_{s}<1 \Rightarrow \alpha_{\mathrm{loc}}=\alpha_{s} & \left\{\begin{array}{l}
\alpha_{s}=\alpha \Rightarrow \text { Family-Vicsek } \\
\alpha_{s} \neq \alpha \Rightarrow \text { intrinsic }
\end{array}\right. \\
\text { if } \alpha_{s}>1 \Rightarrow \alpha_{\mathrm{loc}}=1 & \left\{\begin{array}{l}
\alpha_{s}=\alpha \Rightarrow \text { super-rough } \\
\alpha_{s} \neq \alpha \Rightarrow \text { new class }
\end{array}\right.\end{cases}
$$

In the following we present simulations of a onedimensional growth model that is a nice example of the new dynamics. We have performed numerical simulations of the Sneppen model of self-organized depinning (model $A$ ) [18]. We have found that this model exhibits anomalous roughening of the type described by Eq. (7) for $\alpha_{s}>1$ and $\alpha_{s} \neq \alpha$. In this model the height of the interface $h(i, t)$ is taken to be an integer defined on a onedimensional discrete substrate $i=1, \ldots, L$. A random pinning force $\eta(i, h)$ is associated with each lattice site $[i, h(i)]$. The quenched disorder $\eta(i, h)$ is uniformly distributed in $[0,1]$ and uncorrelated. The growth algorithm is then as follows. At every time step $t$, the site $i_{0}$ with the smallest pinning force is chosen and its height $h\left(i_{0}, t\right)$ is updated $h\left(i_{0}, t+1\right)=h\left(i_{0}, t\right)+1$ provided that the conditions $\left|h\left(i_{0}, t\right)-h\left(i_{0} \pm 1, t\right)\right|<2$ are satisfied. Periodic boundary conditions are assumed. We have studied the behavior of the model in systems of different sizes from $L=2^{6}$ up to $L=2^{13}$. From calculations of the saturated global width $W(L)$ for various system sizes, we find a global roughness exponent $\alpha=1.000 \pm 0.005$ in agreement with previous simulations [18]. We have checked that the scaling of the global width is given by Eq. (1) with a scaling function such as (2). Also in agreement with previous work [18], we find that the time exponent $\alpha / z=0.95 \pm 0.05$. The local width $w(l, t)$ scales as $w(l, t)=t^{\alpha / z} g(l / \xi)$ where the scaling function is given by Eq. (11), and also $\alpha_{\text {loc }}=1$.

From these simulation results, one could conclude that the behavior of the Sneppen growth model is rather trivial and that the exponents $\alpha=\alpha_{\text {loc }}=z=1$ describe its scaling properties. Quite the opposite, this model exhibits no trivial features that can be noticed when the structure factor is calculated. In Fig. 1 we show our numerical results for the structure factor $S(k, t)$ in a system of size $L=2048$. Note that in Fig. 1 the curves $S(k, t)$ for different times are shifted downwards reflecting that $\alpha<\alpha_{s}$. This contrasts with the case of intrinsic anomalous roughening [16,17] where $\alpha_{s}=\alpha_{\text {loc }}$ and $\alpha_{\text {loc }} \leq 1$. The slope of the continuous line is -3.7 and indicates that a new exponent $\alpha_{s}=1.35$ enters the scaling. This can be better appreciated by the data collapse shown in Fig. 2, where one can observe that, instead of being constant, the scaling function $s(u)$ has a negative exponent $u^{-0.7}$ for $u \gg 1$. The exponents used for the data collapse are $\alpha=1, z=1$ and the scaling function obtained is in excellent agreement with Eq. (7) and a spectral exponent $\alpha_{s}=1.35 \pm 0.03$.

The interface in the Sneppen model $A$ is formed by facets with constant slope \pm 1 [18]. The value of the exponents $\alpha=\alpha_{\mathrm{loc}}=1$ and $\alpha_{s}=1.35$ is related to the faceted form of the interface at saturation. It is easy to understand how the anomalous spectral roughness exponent appears due to the faceted form of the interface. For the simpler (and trivial) case of a faceted interface formed by a finite number of identical segments, $N$, of constant slope, $\pm m$, one can show analytically that the

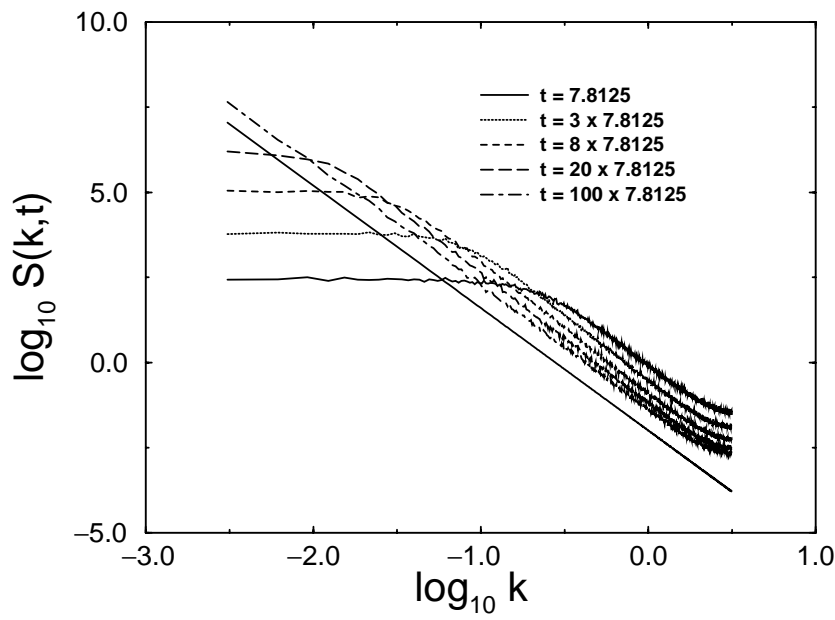

FIG. 1. Structure factor of the Sneppen model for interface depinning at different times. The continuous straight line is a guide to the eye and has a slope -3.7 . Note the anomalous downwards shift of the curves for increasing times. 


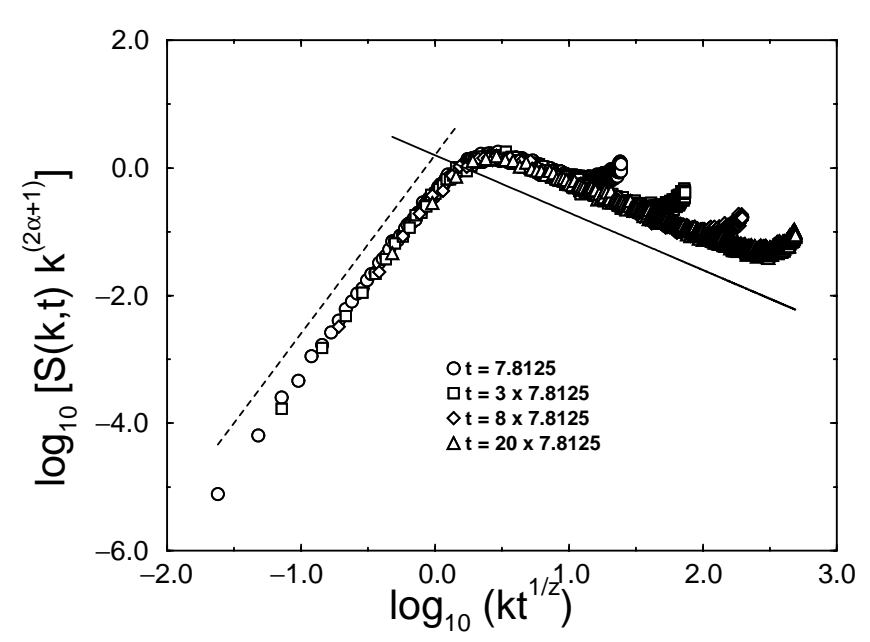

FIG. 2. Data collapse of the graphs in Fig. 1. The exponents used for the collapse are $\alpha=1.0$ and $z=1.0$. The straight lines have slopes -0.7 (solid) and 3.0 (dashed) and are a guide to the eye. The scaling function is given by Eq. (7) with a spectral roughness exponent $\alpha_{s}=1.35 \pm 0.03$. The deviations from the scaling for large values of the argument $k t^{1 / z}$ are due to the finite lattice spacing.

global width $W(L) \sim m^{2} L^{2} / N^{2}$, and the height-height correlation function $G(l) \sim l^{2} m^{2}-N m^{2} l^{3} / L$, which leads to $\alpha=\alpha_{\text {loc }}=1$, while the spectrum $S(k, L) \sim$ $k^{-4} L^{-1}$ as $k \rightarrow 0$. A simple comparison with the anomalous scaling form for the stationary spectrum $S(k, L) \sim k^{-\left(2 \alpha_{s}+1\right)} L^{2\left(\alpha-\alpha_{s}\right)}$ leads to $\alpha_{s}=1.5$. Actually, the facets occurring in the Sneppen model are not formed by identical segments, but rather follow a random distribution [19], which leads to a spectral exponent different from the trivial case.

In summary, we have presented a generic theory of scaling for invariant surface growth. We have shown that the existence of power-law scaling of the correlation functions (scale invariance) does not determine a unique form of the scaling functions involved. This leads to the different dynamic scaling forms recently observed in growth models [4-10] and experiments [11-15] exhibiting anomalous roughening. In particular, interface scale invariance does not necessarily imply Family-Vicsek dynamic scaling. We have derived all the types of scaling (Family-Vicsek, super-rough, and intrinsic anomalous) from a unique scaling ansatz, which is formulated in the Fourier space. The different types of scaling are subclasses of our generic scaling ansatz associated with bounds on the values that the new spectral roughness exponent $\alpha_{s}$ may take. This generalization has allowed us to predict the existence of a new kind of anomalous scaling with interesting features. Simulations of a model for self-organized interface depinning have been shown to be in excellent agreement with the new anomalous dynamics. It has recently been shown [20] that anomalous roughening stems from a nontrivial dynamics of the mean local slopes $\left\langle\overline{(\nabla h)^{2}}\right\rangle$. In contrast, the new anomalous dynamics can be pinned down to growth models in which the stationary state consists of faceted interfaces.

The authors would like to thank R. Cuerno for an earlier collaboration that led to Refs. [16,17]. This work has been supported by the DGES of the Spanish Government (Project No. PB96-0378-C02-02). J. J. R. is supported by the Ministerio de Educación y Cultura (Spain). J. M. L. is supported by a TMR Network of the European Commission (Contract No. FMRXCT980183).

*Electronic address: ramasco@ifca.unican.es

†Electronic address: jmlopez@pil.phys.uniromal.it

[1] A.-L. Barabási and H.E. Stanley, Fractal Concepts in Surface Growth (Cambridge University Press, Cambridge, England, 1995).

[2] J. Krug, Adv. Phys. 46, 139 (1997).

[3] F. Family and T. Vicsek, J. Phys. A 18, L75 (1985).

[4] J. Krug, Phys. Rev. Lett. 72, 2907 (1994).

[5] M. Schroeder et al., Europhys. Lett. 24, 563 (1993).

[6] J. M. López and M. A. Rodriguez, Phys. Rev. E 54, R2189 (1996).

[7] S. Das Sarma et al., Phys. Rev. E 53, 359 (1996).

[8] C. Dasgupta, S. Das Sarma, and J. M. Kim, Phys. Rev. E 54, R4552 (1996).

[9] J. M. López and M. A. Rodriguez, J. Phys. I (France) 7, 1191 (1997).

[10] M. Castro et al., Phys. Rev. E 57, R2491 (1998).

[11] H.-N. Yang, G.-C. Wang, and T.-M. Lu, Phys. Rev. Lett. 73, 2348 (1994).

[12] J. H. Jeffries, J.-K. Zuo, and M. M. Craig, Phys. Rev. Lett. 76, 4931 (1996).

[13] J. M. López and J. Schmittbuhl, Phys. Rev. E 57, 6405 (1998).

[14] S. Morel et al., Phys. Rev. E 58, 6999 (1998).

[15] A. Bru et al., Phys. Rev. Lett. 81, 4008 (1998).

[16] J. M. López, M. A. Rodriguez, and R. Cuerno, Phys. Rev. E 56, 3993 (1997).

[17] J. M. López, M. A. Rodriguez, and R. Cuerno, Physica (Amsterdam) 246A, 329 (1997).

[18] K. Sneppen, Phys. Rev. Lett. 69, 3539 (1992).

[19] J. J. Ramasco, J. M. López, and M. A. Rodriguez (unpublished).

[20] J. M. López, Phys. Rev. Lett. 83, 4594 (1999). 